\title{
Overview of Biosignal Analysis Methods for the Assessment of Stress
}

\author{
I. Ladakis ${ }^{1 *}$, I. Chouvarda ${ }^{1 *}$ \\ ${ }^{I}$ Lab of Computing, Medical Informatics and Biomedical Imaging Technologies, Aristotle University of Thessaloniki, Thessaloniki, Greece
}

\begin{abstract}
Objectives: Stress is a normal reaction of the human organism induced in situations that demand a level of activation. This reaction has both positive and negative impact on the life of each individual. Thus, the problem of stress management is vital for the maintenance of a person's psychological balance. This paper aims at the brief presentation of stress definition and various factors that can lead to augmented stress levels. Moreover, a brief synopsis of biosignals that are used for the detection and categorization of stress and their analysis is presented. Methods: Several studies, articles and reviews were included after literature research. The main questions of the research were: the most important and widely used physiological signals for stress detection/ assessment, the analysis methods for their manipulation and the implementation of signal analysis for stress detection/assessment in various developed systems. Findings: The main conclusion is that current researching approaches lead to more sophisticated methods of analysis and more accurate systems of stress detection and assessment. However, the lack of a concrete framework towards stress detection and assessment remains a great challenge for the research community.
\end{abstract}

\begin{tabular}{|c|c|c|c|}
\hline \multicolumn{4}{|l|}{ Keywords: } \\
\hline \multicolumn{4}{|l|}{ Stress; } \\
\hline \multicolumn{4}{|l|}{ Biosignals; } \\
\hline \multicolumn{4}{|l|}{ Analysis; } \\
\hline \multicolumn{4}{|c|}{ Stress Detection; } \\
\hline \multicolumn{4}{|c|}{ Stress Assessment. } \\
\hline \multicolumn{4}{|c|}{ Article History: } \\
\hline Received: & 26 & January & 2021 \\
\hline Revised: & 17 & March & 2021 \\
\hline Accepted: & 23 & March & 2021 \\
\hline Published: & 01 & April & 2021 \\
\hline
\end{tabular}

\section{1- Introduction}

Stress [1] is referred to as a normal reaction of the organism to anything that threatens its homeostasis [2]. Any such threat is characterized as a stressor, i.e., a factor that induces the stress reaction. Therefore, psychological stress can be defined as a series of behavioral, mental, and physiological reactions (stress responses) caused by exposure on stressors or environmental resolution requests [3]. On the other hand, mental stress is referred as a form of stress that depends on a person's perception of environmental events, inducing distress or anxiety experiences [4]. Elliot and Eisdorfer [5] suggest a taxonomy to characterize these situations in which a person can be involved during his/her lifetime. The distinction they propose takes under consideration two important aspects: duration and course. Thus, the five types of stress in terms of time lapse are [6]:

1) Acute time-limited stress: include challenges like public speaking or mental arithmetic.

2) Brief naturalistic stress: situations that demand confrontation with a real-life short-term challenge like the participation on academic procedures.

3) Stressful event sequences: events that induce mourning and a series of reactions, such as the loss of a dear person.

4) Chronic stress: situations that encircle a person and affect his life indirectly, eventually forcing him to change behaviors or the way he perceives himself. Such situation is a traumatic experience or the daily confrontation of oppressive manners in workplace.

*CONTACT: Iladakig@auth.gr; Ioannach@auth.gr

DOI: http://dx.doi.org/10.28991/esj-2021-01267

(C) 2021 by the authors. Licensee ESJ, Italy. This is an open access article under the terms and conditions of the Creative Commons Attribution (CC-BY) license (https://creativecommons.org/licenses/by/4.0/). 
5) Distant stress: traumatic experiences that occurred in the past but maintain the potential to affect a person's immune system function. Such examples are rape, to be prisoner of war, to witness a catastrophic event.

Stressor is called any stimulus that induces the release of stress hormones. The most common categories of stressors are [7]:

1) Physiological or physical stressors that include injuries, environmental conditions, pain, fatigue etc.

2) Psychological stressors that could be the result of mourning, missed deadlines, alterations in habits or in social life and negative situations in general.

While a stressor relates to the cause, "stress responses" are the physical and mental changes induced by stressors, which can be observed in a qualitative and quantitative manner to recognize the level of stress.

Increased stress is a phenomenon of the modern world. It is directly linked to the rapid increase in the rhythms of life, the constant change in the way modern people perceive themselves within society, but also all the reality around them, the constant demand for specialization and competitive qualifications in the field of the profession, as well as the very way in which the individual has developed his/her values, ideals and perception of himself/herself, which often contradicts and conflicts with reality. Various stressful situations have been reported in the literature [8], psychological and biological, reflecting the range of environmental stimuli that can increase stress levels. Among others, work stress is referred as widely observed in workplaces ([9-11]) leading employers to implement intervention techniques for the reduction of its negative impact [12-14].

Even though temporary stress is a normal reaction inherent in human everyday life, incorrect management of increased stress can lead to a decrease in its functionality during its interaction with the world or to major health problems [15], potentially having a permanent impact on a person's life. Due to the extent of the phenomenon and the effect it has on modern society, it is necessary to outline ways of dealing with or managing it. This is the reason why research community has tried over the years to develop methods capable to estimate stress level accurately. The most common or widely used method is by means of questionnaires. Nevertheless, this method has various disadvantages, basically its subjectivity and the time it demands. An accurate, real-time and objective approach in the direction of stress detection has been considered of vital importance.

Measurements of various localized biomarkers already constitute a key part in the detection and evaluation of stress. These biomarkers are actually normal biological signals that can express change in the normal functioning of the body. Recent research works and studies investigate various physiological signals about their capability to act as satisfactory stress indicators taking under consideration the diversity of stressors and their impact to each individual. Both the collection of biosignals and their qualitative evaluation and categorization are necessary and interrelated procedures in the direction of drawing safe conclusions and making appropriate moves towards stress management.

In the following sections a brief summary of physiological signals that have been used as stress biomarkers will be presented followed by the elaboration of analysis methods and specific features that indicate changes at stress levels, and an overview of relevant applications. Specifically, the main body of the paper consists of four sections. Section 2 refers to the physiological signals that can be used as biomarkers of stress and to the analysis methods that have been used towards the exploitation of these signals for feature extraction and prediction or assessment of stress levels. Various research studies and applications that attempt to evaluate and manage stress levels are presented in section 3 . Finally, the last two sections of the paper are summing up the findings of the attempted overview and proceed to the extraction of conclusions. The work flow, the number of studies per section and the criteria of inclusion are described in figure 1, while figure 2 presents a diagram regarding the number of included studies per year. The last diagram depicts the authors' attempt to include recent studies (most of them have been published after 2015).

\section{2- Overview of Signals and Methods}

\section{2-1-Physiological Signals - Biomarkers of Stress}

A number of physiological signals have been tested for their association with increased stress, either isolated or in combination with others, such as electrocardiogram (ECG), electromyogram (EMG), galvanic skin response (GSR), blood pressure (BP), skin temperature (SKT), blood volume pulse (BVP), respiratory inductance plethysmogram (RIP), Photoplethysmogram (PPG), electrodermal activity (EDA) and electroencephalogram (EEG). These signals have been processed in various ways and analyzed with statistical and machine learning methods.

Detailed description of the physiological signals' characteristics or basic information used to detect stress from the aspect of computer science is provided in the existing literature [16-19]. The following subsections describe signals representing different physiological phenomena that can be used as biomarkers of stress and specific information that can be extracted from them after standard analysis. 


\section{Study Diagram}

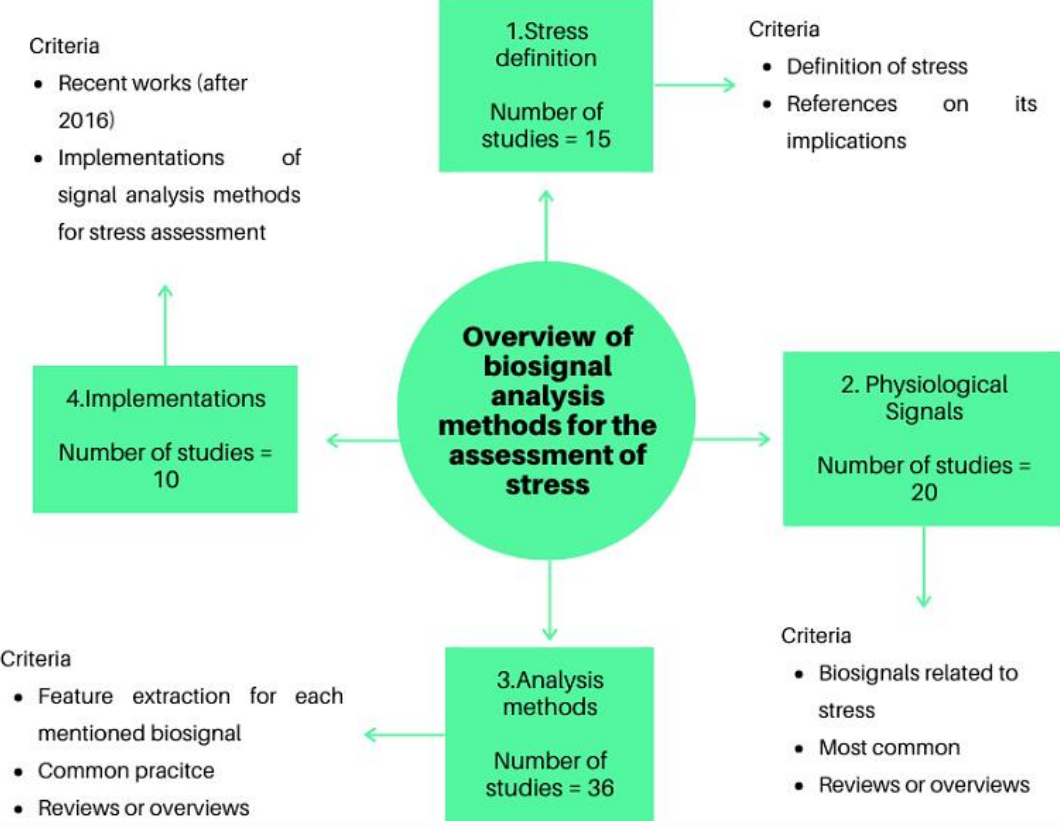

Figure 1. Study diagram.

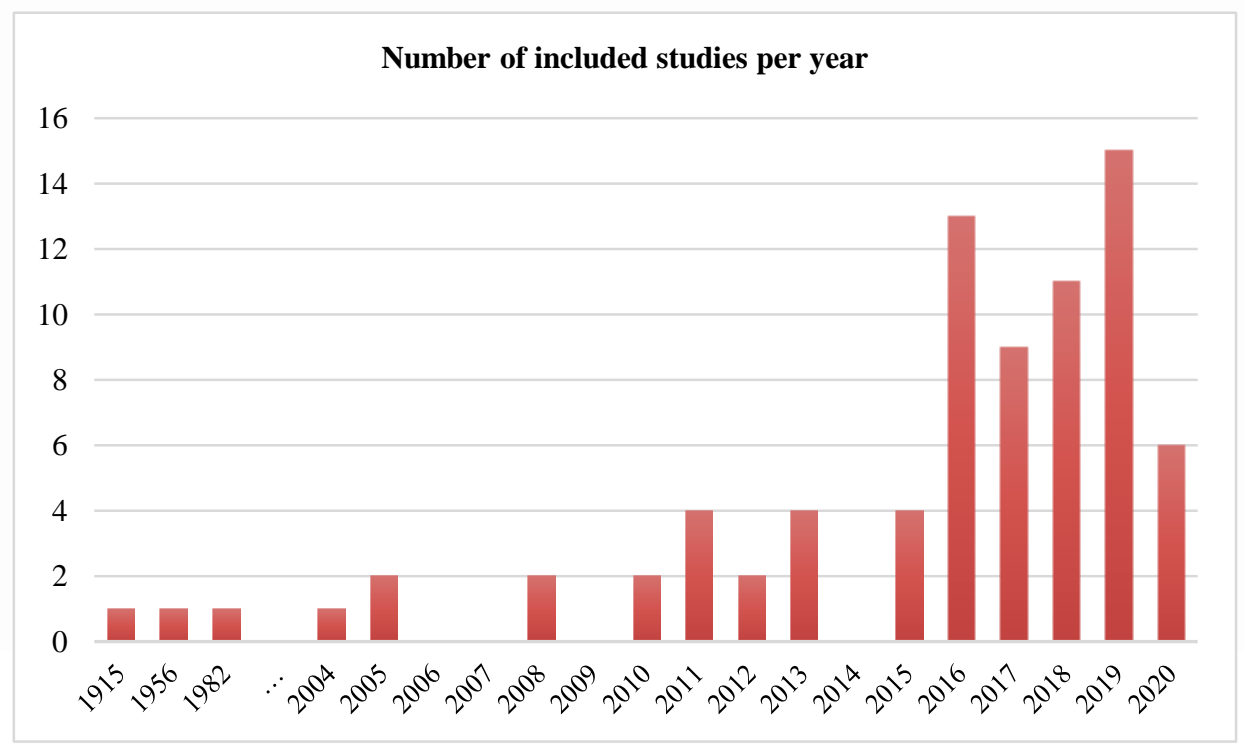

Figure 2. Number of included studies per year.

\section{Electrodermal Activity}

Electrodermal activity (EDA) sensors measure changes in electrical conductivity on the surface of the skin [18]. Such changes may be caused by various physical/event or emotional stimuli that cause a variety of sweat secretion reactions. Due to signal control only by the sympathetic nervous system, it becomes an ideal signal for measuring the increase or level of stress. It varies depending on gender, nutrition, skin type and situation.

Galvanic Skin Response (GSR) indicate skin electrical resistance capturing the conductivity of skin. The GSR signal is referred as a reliable biomarker of stress [20] and can be distinguished into two different elements/components:

- The level of conductivity of the skin (skin conductance level - SCL): component with slow changes, represents average skin conductivity in a period of time. It is a measure of psychophysiological activation.

- The conductivity response of the skin (skin conductance response - SCR): component with quick changes, appears in relation to a single stimulus. It is a measure of reactions to sudden stimuli.

Any sudden stimulus can induce variations to skin's resistance. During exposure at stressors the sweat glands are activated and, as a consequence, skin conductance is augmented. That makes GSR an ideal measure for sympathetic activation and stress detection. 


\section{Cardiovascular System Activity}

In a situation of acute stress occurrence, the sympathetic nervous system increases heart rate, respiration activity, electrodermal activity etc. When the individual has recovered from stress, the parasympathetic nervous system reverses the stress impact. Knowing that heart is controlled by the autonomic nervous system, cardiac activity can provide vital information about stress levels via the assessment of autonomic nervous system's state.

It can be measured through various physiological signals, with the most important being ECG, BVP and PPG. BVP reflects the amount of blood vessels during a certain time interval and is an indicator of blood flow. Under stress, BVP decreases while the opposite occurs in a calm state [21]. The electrocardiogram can easily calculate the heart rate (HR) or even the diversity of the heart rate (HRV), which demonstrates the variability of the heart rate, i.e., depicts the beatto-beat variation in the R-R interval. As with electrodermal activity, cardiovascular activity measurements must be accompanied by information on the user's emotional ability to react in order to distinguish positive and negative impact situations. HR alone is not sufficient to measure and evaluate stress [22] and an attempt to turn into other ECG derived features for more consistent information is observed at least the last decade, even though several studies in the existing literature imply the opposite [23]. On the other hand, various research works have been using HRV alone or in conjunction with other physiological signals with very promising results regarding stress detection [22, 24].

\section{Muscle Activity}

It is measured through electromyograms with appropriate electrode placement. Through the appropriate categorization and detection of the activity of specific muscle groups, such as cheekbones, forehead, mouth or cheeks, a conclusion can be drawn about the psychological state of the person, as well as his level of stress or satisfaction [25, 26]. However, it is not the safest method to draw conclusions and it is proposed to use it as an additional marker [27].

\section{Respiratory System Activity}

Breathing is inextricably linked to cardiovascular activity and is mainly affected by changes between calm and stress situations (surprise, joy, sadness, stress). The breathing rate and width of breathing are the two measurements mainly used to assess respiratory activity. However, it is reported that breathing rate is among the least sensitive metrics of respiratory data analysis. Although stress often induces increase in respiratory rate, the information of respiratory signal (RSP) should be used as supplementary [23].

\section{Brain Activity}

Electroencephalogram (EEG) is the most common signal that provides information about brain activity. It depicts quantified brain functions and conditions. Although it is a rather complex signal that demands careful and thorough processing, it has been widely studied due to the easiness of its collection (non - invasive methods), its supreme temporal resolution and the low set-up cost [28]. One of its advantages is its ability to capture alterations in cognitive activity within millisecond scale due to its resolution.

There are four categories of EEG signal based on frequency bands: Delta $(0.5-4 \mathrm{~Hz})$, Theta $(4-8 \mathrm{~Hz})$, Alpha $(8-$ $13 \mathrm{~Hz})$ and Beta $(14-30 \mathrm{~Hz})$. It has been reported that EEG power spectrum features present significant correlation with stress levels $[29,30]$. Additionally, there are references that state the existence of many similarities between stress state and negative emotion state [31]. Subhani, Xia and Malik [32] present a categorization in relation to stress levels that refers to the impact of stress in each frequency band of the signal. Delta's increased power seems to reflect inclusion of the individual into difficult situations. Theta's increased power indicate stressed state as well as alpha's suppressed power. Finally, beta's power varies depending on task difficulty. EEG signal has been widely used in systems aiming to detect, evaluate and/or reduce stress [33, 34]. Most of these systems aim at the detection of acute stress, but there are some efforts investigating chronic stress [35]. The rapid technological advancements lead to an increasing production of commercially available EEG headsets at reasonable costs, a fact that could result to the increase of EEG attractiveness as stress indicator.

\section{2-2-Signal Analysis Methods for Stress Level Recognition}

Most of the abovementioned signals need at least some basic processing before they can provide any information for stress detection or mapping. This information can be used as inputs in machine learning techniques and approaches that are widely studied nowadays and are quite promising at predicting stress levels under certain conditions or situations. A brief summary of the processing methods applied to the most relevant signals with regard to stress levels is presented below, as well as some of the machine learning techniques mentioned in the existing literature. It should be mentioned that the accuracy or the reliability of each feature extracted by any analysis is still studied and analyzed in most approaches via statistical analysis.

According to Yu et al. (2018) [22] the most reliable signals that better capture stress levels are HRV and GSR, while RSP is regarded as useful supporting signal [29]. However, numerous studies indicate EEG signal as a valuable measurement in monitoring and evaluating stress levels. The featuring of these four signals will be discussed below. 


\section{Feature Extraction}

HRV [36, 37]: HRV can be categorized in $24 \mathrm{~h}$, short term $(\sim 5 \mathrm{~min})$ or brief and ultra-short term $(<5 \mathrm{~min}) .24 \mathrm{~h}$ HRV recordings are reported to have higher predictive capability that short-term measurements [38]. Its analysis is usually categorized into time-domain, spectral-domain and non-linear analysis Time-domain analysis is implemented at HRV measurements that may ranges from less than 1 minute and more than 24 hours. The features extracted and evaluated via time-domain analysis are:

- Mean heart rate (HR);

- Mean heartbeat interval (RR);

- Standard deviation of R-R intervals between normal beats (SDNN);

- Root mean square of the difference between successive R-R intervals (RMSSD);

- The percentage of heartbeat intervals with a difference in successive heartbeat intervals greater than 50 milliseconds (pNN50).

On the other hand, the features extracted via spectral-domain analysis, in which Fast Fourier Transform is usually applied, are:

- An ultra-low-frequency component that extracted from a recording period of at 24 hours and more (ULF, $<0.003$ $\mathrm{Hz}$ ). There is no clear evidence about its impact on stress;

- A very low-frequency component with measurement time requirement of at least 5 minutes (VLF, $0.0033-0.04$ $\mathrm{Hz}$;

- A low-frequency component that is mediated by both sympathetic and parasympathetic nervous system and is typically recorded over a minimum of 2 minutes period (LF, $0.04-0.15 \mathrm{~Hz}$ );

- A high-frequency component mediated by the parasympathetic nervous system (HF, $0.15-0.4 \mathrm{~Hz})$. This component is recorded over a minimum of 1 minute period. Low LF power correlates with stress or worry;

- LF to HF ratio that is used as an index of autonomic balance (LF/HF) and is based on 24-hour long measurements;

- Finally, non-linear measurements depict the unpredictability of a time series which is induced by the very complexity of HRV signal. The features extracted from non-linear analysis are categorized in the invariant and informational domains [39]. Specifically, invariant domain includes features like Detrended Fluctuation Analysis with a1 and a 2 components, Fractal Dimension, Hurst Exponent, Largest Lyapunov Exponent and Correlation Dimension, while Shaffer and Ginsberg also add Poincaré plot [37];

- Informational domain includes features like Approximate Entropy, Sample Entropy, Shannon Entropy and Multiscale Entropy.

Non-linear analysis methods have not been widely implemented in the direction of stress detection yet. Lower RMSSD values and HF power and higher LF power are reported to correlate with increased stress levels [40, 41]. Lastly, useful packages for HRV analysis have been developed, such as RHRV package for R programming language [42], that provide integrated analysis solutions to data scientists and researchers.

GSR [43, 44]: this signal is usually captured in hands or feet due to the high density of sweat glands. Its analysis contains the distinction between its slowly altering part (SCL) and its fast-altering part (SCR). The features that are commonly extracted from these two components of the signal are the amplitude and the latency of SCR and the average SCL value. Lee and Kleinsmith [45] in their experiments pointed out the most discriminative statistical features of GSR for stress detection, which are: the mean GSR, the number of peaks and the max peak amplitude, applied to the components of the signal. GSR normally needs some preprocessing before the stage of feature extraction, such as downsampling, low-pass filtering, moving average and normalization for artifact removal [46]. Braithwaite, Watson, Jones and Rowe [47] present an integrated guide towards techniques for analyzing GSR based on AcqKnowledge software. Other similar software for GSR/EDA signal analysis in cvxEDA [48] and ledalab [49, 50]. Some of the reported analysis methods are Continuous Decomposition Analysis, Discrete Deconvolution Analysis, Tonic/phasic Decomposition, Spectral Analysis, Point Process and State-Space process. The analysis methods proposed are working mainly on the decomposition of the two GSR components and then at the restoration, detection, and thorough scoring of their features.

RSP: Respiratory rate or respiratory frequency is a rather neglected signal, whose importance is designated nowadays with the recent researching advantages in its understanding [51]. Some analyses refer to the detection of peaks and valleys in RSP signal that mirror the inspiration and expiration phase of breathing respectively [52]. Massaroni Nicolò, Sacchetti and Schena [53] in their review concentrate various approaches of collecting and analyzing RSP signal via different sensor technologies focusing on contactless measuring. RSP signal is mentioned to be considered a supplementary source of information about stress levels, used in combination with other signals like HRV [54]. Some 
applied analysis methods are filtering (band-pass or low-pass), down-sampling and various peaked-conditioned approaches. Some of the available analysis packages and programs are respR [55] and Nellcor Respiration Rate Software.

EEG [23]: The most typical analysis, although not the only one, in EEG signal is to accurately estimate the signal components present in each of the five different frequency bands via frequency-domain analysis techniques. This issue could be a rising challenge basically because of the need for cautious removal and cleaning of the signal before any analysis implementation or feature extraction [56]. This issue has attracted the interest of the researchers and various solutions have been proposed over the years $[57,58]$. The analysis methods for band's feature extraction are the following:

- Short-term Fourier transform (STFT) in which the separation of stationary signals is carried out into small fragments. This technique provides results that are better located in time than Fourier transform (FT) technique which is unsuitable for non-stationary signal analysis. Some features extracted from this analysis total energy of band, maximum value of band, relative power, spectral entropy, frontal asymmetry and event-related (de-) synchronization which is inversely related to band power.

- Wavelet transform (WT) that is a better suited technique for analysis of sudden short duration signal alterations.

- Singular Value Deconvolution (SVD) for the examination of singular spectrum. A feature extracted from this analysis is Singular Value Deconvolution Entropy [59].

There are various available software programs or packages, open source or commercial, for EEG signal analysis, such as PyEEG [60], eegkit package for R [61] and BIOPAC.

\section{Machine Learning (ML) Techniques}

Most of the existing work implementing machine learning algorithms for the detection and prediction of stress use HRV or EDA/GSR signal as their collection does not demand intrusive methods and they provide relatively solid information about stress levels [62]. Shatte et al. (2019) [63] provide in their review an extensive list of machine learning techniques used for stress detection, prediction or assessment. Another interesting study [64] presents a brief overview of biosignals used in stress detection as well as machine learning classifiers used to characterize stress levels. An extensive review was devised by Elzeiny and Qaraqe [65] reporting on ML techniques implemented for the automated detection of stress and the identification of stimulus. An interesting aspect that is revealed in this work is the different classification approaches. Depending on the application, the target group or the selected features (input to machine learning algorithms), the stress levels are distinguished either with a binary logic (stress or no stress) or in several (more than two) levels. The ambiguity of these approaches reflects the diversity of the desired targets of each system as well as the biased direction induced by the way the research teams define stress.

There are two ways for a machine to learn: supervised and unsupervised learning. The difference between the pattern recognition technique of the two approaches is whether the input data is labeled (supervised) or not (unsupervised). Models of both approaches have been reported in literature regarding stress recognition.

The supervised model of support-vector machine (SVM) has been implemented in various studies as well as logistic regression (LR), using as training data mainly HRV signal that was obtained either from databases of clinical data or from commercial sensors used integrated in the systems [66-69]. Elgendi and Menon [70] used both unsupervised and supervised ML techniques to assess stress, getting the result that ECG signal marks as the most reliable biomarker of stress among the tested signals (EMG, GSR, HR, RSP). An interest fact about this work is the combination of three unsupervised ML approaches, namely principal component analysis, connectivity-based clustering and K-means clustering.

Specifically, the most common ML methods used in signal classification for stress detection or assessment are [71]:

- Logistic Regression (LR) where the probability of the classification in stress or rest state is determined by weighed features;

- Support-Vector Machine (SVM) that examines the existence of an optimal hyperplane to distinct the input data based on selected analysis features;

- Decision Trees (DT) that are based on hierarchical partitioning. The algorithm moves along the nodes' paths that represent observations about a certain item to conclude its target value. This technique is very attractive because of its intelligibility and simplicity;

- Random Forests (RF) that constitute a combination of DT where each tree is modulated using a random selection of data and features;

- Bayesian Networks (BN) that are directed acyclic graphs with nodes that embody variables like features and stress levels and edges depicting direct correlations between the nodes. BN can be static or dynamic. 
Other machine learning techniques reported are k-Nearest Neighbours (kNN), Linear Discriminant Analysis (LDA), Hidden Markov Model (HMM) and Artificial Neural Network (ANN) [64].

\section{3- Implementations of the Analysis Methods}

Various approaches are reported for the management and process of physiological signals that could be biomarkers of stress. Hou et al. (2015) [31] present a methodology/algorithm for extracting specific characteristics from the EEG to detect three conditions: emotional charge, mental overload and stress detection/monitoring. They use modern technology of portable encephalogram devices and existing literature to associate specific characteristics of the EEG with specific situations, but without giving clear answers. The system is completed by developing software to view and analyze the detected levels of each of the intended mental/psychological situations. In the above work, an experiment was conducted to identify stress to study the association between overload, emotion and stress. Based on the results of the experiment, they propose a new approach to stress detection that uses the idea of combining recognized states of emotion and overload. Depending on the category to which these two situations fall, a conclusion is drawn about the level of stress in the subject. It is then followed by a corresponding visualization in software developed for the purposes of research.

Nakashima et al. (2015) [72] attempt to form a framework for detecting stress during daily work activity. In their experiment they try to combine the extracted characteristics of various physiological signals from commercial portable sensors and discuss their ability to draw safe and effective conclusions. Stroop Test was used as a method of stress stimulation, and in addition to the BVP and EDA physiological signal collection sensors, pressure distribution sensors (in the seat, back and legs) and an eye position detection sensor were used. The conclusions state that indeed the combination of a series of physiological signals contributes to the formation of a fuller picture of the state of stress, even if the correlation between them and/or the most appropriate combination has not been systematically tested.

Sriramprakash et al. (2017) [73] conducted a study in an effort to determine the best possible categorization and the best possible characteristic of physiological signals for stress detection in workers. The study's measurements focused on collecting signals related to cardiovascular and electrodermal activity. Their conclusions suggest that the time and frequency analysis of HR, HRV and GSR can extract specific characteristics and information that are sufficient to predict stress in the working environment. Cho et al. (2017) [74] attempted to implement a Kernel-Based Extreme Learning Machine analysis technique from PPG, EDA, SKT signals collected during experiments and after analysis. This analysis includes the detection of peak features and HRV derivatives from PPG. Their machine learning algorithm aimed at the correct classification of the collected signals in five different categories that indicated stress levels. After a stress inducing experiment, they concluded that the selected biosignals, their processing methods and the machine learning algorithm gave optimistic results about the precise detection and classification of stress levels. Minguillon et al. (2018) [75] proposed the collection and statistical analysis of a multitude of signals for stress level characterization (EEG, ECG, EMG, GSR) summarizing the existing literature.

Particularly notable is the approach presented by Borthakur (2020) [19] on the analysis of cardiac and respiratory signals with the aim of optimized stress level characterization. The approach focused mainly at the process of HRV signal: the standard deviation of NN intervals, low frequency power and high frequency power were analyzed implementing time domain and frequency domain analysis. Moreover, thorough statistical analysis was performed to examine the level of correlation between the analyzed signals and their capability to indicate stress. A similar work [76] describes a process of analyzing and extracting features of PPG and EDA signals, while developing a machine learning algorithm to categorize the stress levels of the participants in the experiment. Time domain and frequency domain features of HRV and EDA were calculated underlining the significance of these two signals in the attempt to detect and characterize stress.

Another interesting work presents a power spectrum analysis and heatmap topology approach as a processing method for EEG signal in order to capture neural activity before and after a sustainable relaxation technique. The analysis focuses on the observed changes in brain rhythms [77]. A number of activation exercises were implemented at the experiment phase to observe and better validate the extracted conclusions. EEG and EDA signals were also analyzed in the work of Saitis and Kalimeri [59] aiming at the classification of stressful environments. For EEG power and singular spectrum analyses were performed for the extraction of specific features like relative power, spectral entropy, and frontal asymmetry. A deconvolution method was implemented for EDA signal to distinct between tonic and phasic components that were further analyzed. Finally, EDA signal was also examined in the work of Siirtola [78] that aimed at continuous stress detection with the use of a wearable commercial sensor. The classification of stress levels was based on a machine learning algorithm that used an open data set to be trained.

\section{4- Results and Discussion}

In general, the proposed approaches and the methods developed revolve around specific physiological signals. The discussion is more about finding the best way to manage them to extract characteristics that can give accurate information about stress levels and characterize them with corresponding accuracy. Ultimately, the authors, through the 
results of the review, suggest that one of the best ways to measure and evaluate stress status is to collect and analyze a combination of physiological signals, as well as that the most appropriate physiological signals for stress determination are the extracted signals of ECG, confidently improving the identification level by collecting and analyzing additional signals. The most common combination of biosignals is HR, HRV and RSP, with an increasing interest towards the inclusion of GSR/EDA and its combination with ECG derived features like HRV. The last seems to have attracted the attention of research community and various metrics have been studied regarding their relation with stress levels. EEG signal has also been widely studied and implemented for stress recognition, but its combination with other signals is limited because of the challenging acquisition in daily life environments. However, there is no definite result that indicates the existence of a gold standard combination of biosignals. Machine learning techniques are applied to categorize stress levels based on previous signal analysis, while for most systems the selection of reference signals is made having in mind the need for easier and more immediate implementation in real life.

\section{5- Conclusion}

The overviewed field has proven challenging and demanding, studied for years, and yet lacking a robust, concrete framework. The technological advance and the development of increasingly sophisticated processing methods and algorithms, due to the advance of computational intelligence and power, allow the appearance of novel approaches investigating the best way to manage the stress detection/evaluation issue. Important aspects of stress should be clearly defined and stated on future works, such as the aim of each approach regarding acute or chronic stress, the classification of stress levels and the declaration of baselines towards the better categorization of stress, in order to enhance the detection/prediction accuracy of the developed systems and result in products capable to adequately manage the problems induced by stress in modern world. The present overview depicts the vast extent of research works existing in this field and the promising effort towards the development of systems and frameworks aiming at the wellbeing of modern people.

\section{6- Abbreviations}

\begin{tabular}{|c|c|c|c|c|c|}
\hline ANN & Artificial Neural Network & GSR & Galvanic Skin Response & ML & Machine Learning \\
\hline BN & Bayesian Networks & HF & High Frequency & pNN50 & $\begin{array}{l}\text { percentage of adjacent NN intervals that } \\
\text { differ more than } 50 \mathrm{~ms}\end{array}$ \\
\hline BP & Blood Pressure & HMM & Hidden Markov Model & PPG & Photoplethysmogram \\
\hline BVP & Blood Volume Pulse & HR & Heart Rate & RF & Random Forest \\
\hline DT & Decision Trees & HRV & Heart Rate Variability & RIP & Respiratory Inductance Plethysmogram \\
\hline ECG & Electrocardiogram & IBI & Inter Beat Interval & RMSSD & $\begin{array}{l}\text { Root Mean Square of the Successive } \\
\text { Differences }\end{array}$ \\
\hline EDA & Electrodermal Activity & kNN & k-Nearest Neighbours & RSP & Respiratory Signal \\
\hline EEG & Electroencephalogram & LDA & Linear Discriminant Analysis & SCL & Skin Conductance Level \\
\hline EMG & Electromyogram & $\mathbf{L F}$ & Low Frequency & SCR & Skin Conductance Response \\
\hline FT & Fourier Transform & LR & Logistic Regression & SDNN & Standard Deviation of NN intervals \\
\hline SKT & Skin Temperature & STFT & Short-Term Fourier Transform & SVD & Singular Value Deconvolution \\
\hline SVM & Support-Vector Machine & ULF & Ultra-Low-Frequency & VLF & Very Low-Frequency \\
\hline WT & Wavelet Transform & & & & \\
\hline
\end{tabular}

\section{7- Declarations}

\section{7-1-Author Contributions}

Conceptualization, I.L. and I.C.; methodology, I.L.; formal analysis, I.L.; investigation, I.L.; resources, I.L.; writing - original draft preparation, I.L.; writing-review and editing, I.L. and I.C.; supervision, I.C.; project administration, I.C.; funding acquisition, I.C. All authors have read and agreed to the published version of the manuscript.

\section{7-2-Data Availability Statement}

No new data were created or analyzed in this study. Data sharing is not applicable to this article.

\section{7-3- Funding}

The authors received no financial support for the research, authorship, and/or publication of this article. 


\section{7-4- Conflicts of Interest}

The author declares that there is no conflict of interests regarding the publication of this manuscript. In addition, the ethical issues, including plagiarism, informed consent, misconduct, data fabrication and/or falsification, double publication and/or submission, and redundancies have been completely observed by the authors.

\section{8- References}

[1] Selye, Hans. "The Stress of Life”, McGraw-Hill Book Company, United States (1956).

[2] Cannon, W. "Bodily Changes in Pain, Hunger, Fear and Rage. Ed.” Appleton \& Company, (1915).

[3] Suzuki, Shin-ichi, and Daisuke Ito. "Psychological Stress." Encyclopedia of Behavioral Medicine (2013): 1561-1561. doi:10.1007/978-1-4419-1005-9_421.

[4] Salomon K. "Mental Stress. In: Gellman M.D., Turner J.R. (eds) Encyclopedia of Behavioral Medicine. Springer, New York, NY, (2013).

[5] Elliot, Glenn R., and Carl Eisdorfer. "Stress and human health: An analysis and implications of research. A study by the Institute of Medicine." National Academy of Sciences 79 (1982): 11-24.

[6] Segerstrom, Suzanne C., and Gregory E. Miller. "Psychological Stress and the Human Immune System: A Meta-Analytic Study of 30 Years of Inquiry.” Psychological Bulletin 130, no. 4 (2004): 601-630. doi:10.1037/0033-2909.130.4.601.

[7] Elzeiny, Sami, and Marwa Qaraqe. "Blueprint to workplace stress detection approaches." In 2018 International Conference on Computer and Applications (ICCA), IEEE (2018): 407-412. doi:10.1109/COMAPP.2018.8460293.

[8] Schneiderman, Neil, Gail Ironson, and Scott D. Siegel. "Stress and health: psychological, behavioral, and biological determinants." Annual review of clinical psychology 1 (2005). doi:10.1146/annurev.clinpsy.1.102803.144141.

[9] The Workplace and Health. 2016. The workplace and health. The Robert Wood Johnson Foundation. Available online: http://www.rwjf.org/en/library/research/2016/07/the-workplace-and-health.html (accessed on 26 December 2020).

[10] Hassard, Juliet, Kevin RH Teoh, Gintare Visockaite, Philip Dewe, and Tom Cox. "The cost of work-related stress to society: A systematic review." Journal of occupational health psychology 23, no. 1 (2018): 1-17. doi:10.1037/ocp0000069.

[11] Zawadzki, Matthew J., Stacey B. Scott, David M. Almeida, Stephanie T. Lanza, David E. Conroy, Martin J. Sliwinski, Jinhyuk Kim, et al. "Understanding Stress Reports in Daily Life: a Coordinated Analysis of Factors Associated with the Frequency of Reporting Stress.” Journal of Behavioral Medicine 42, no. 3 (January 1, 2019): 545-560. doi:10.1007/s10865-018-00008-x.

[12] Ganster, Daniel C., and Christopher C. Rosen. "Work Stress and Employee Health.” Journal of Management 39, no. 5 (February 19, 2013): 1085-1122. doi:10.1177/0149206313475815.

[13] Gladwell, Valerie, and D K Brown. "Green Exercise in the Workplace." Green Exercise: Linking Nature, Health and WellBeing, (2016): 139-49.

[14] Klatt, Maryanna, Chris Norre, Brenda Reader, Laura Yodice, and Susan White. "Mindfulness in motion: A mindfulness-based intervention to reduce stress and enhance quality of sleep in Scandinavian employees." Mindfulness 8, no. 2 (2017): $481-488$. doi:10.1007/s12671-016-0621-x.

[15] Yaribeygi, Habib, Yunes Panahi, Hedayat Sahraei, Thomas P. Johnston, and Amirhossein Sahebkar. "The impact of stress on body function: A review." EXCLI journal 16 (2017): 1057-1072. doi:10.17179/excli2017-480.

[16] Sioni, Riccardo, and Luca Chittaro. "Stress detection using physiological sensors." Computer 48, no. 10 (2015): 26-33. doi:10.1109/MC.2015.316.

[17] Kyriakou, Kalliopi, Bernd Resch, Günther Sagl, Andreas Petutschnig, Christian Werner, David Niederseer, Michael Liedlgruber, Frank H. Wilhelm, Tess Osborne, and Jessica Pykett. "Detecting moments of stress from measurements of wearable physiological sensors." Sensors 19, no. 17 (2019): 3805. doi:10.3390/s19173805.

[18] Goumopoulos, C., and E. Menti. "Stress Detection in Seniors Using Biosensors and Psychometric Tests." Procedia Computer Science 152 (2019): 18-27. doi:10.1016/j.procs.2019.05.022.

[19] Borthakur, Debanjan. "Cardiorespiratory Optimized Guided-Breathing for Post-Stress Recovery in a Group Setting." PhD diss., McMaster University, (2020).

[20] Bakker, Jorn, Mykola Pechenizkiy, and Natalia Sidorova. "What's your current stress level? Detection of stress patterns from GSR sensor data." In 2011 IEEE 11th international conference on data mining workshops, IEEE, (2011): 573-580. doi:10.1109/ICDMW.2011.178.

[21] Zhang, Bo, Yann Morère, Loïc Sieler, Cécile Langlet, Benoît Bolmont, and Guy Bourhis. "Stress Recognition from Heterogeneous Data.” Journal of Image and Graphics 4, no. 2 (2016): 116-121. doi:10.18178/joig.4.2.116-121. 
[22] Yu, Bin, Mathias Funk, Jun Hu, Qi Wang, and Loe Feijs. "Biofeedback for Everyday Stress Management: A Systematic Review." Frontiers in ICT 5 (September 7, 2018). doi:10.3389/fict.2018.00023.

[23] Giannakakis, Giorgos, Dimitris Grigoriadis, Katerina Giannakaki, Olympia Simantiraki, Alexandros Roniotis, and Manolis Tsiknakis. "Review on psychological stress detection using biosignals." IEEE Transactions on Affective Computing (2019): 122. doi:10.1109/TAFFC.2019.2927337.

[24] Huysmans, Dorien, Elena Smets, Walter De Raedt, Chris Van Hoof, Katleen Bogaerts, Ilse Van Diest, and Denis Helic. "Unsupervised learning for mental stress detection-exploration of self-organizing maps." Proc. of Biosignals 20184 (2018): 2635. doi:10.5220/0006541100260035.

[25] Westerink, Joyce HDM, Egon L. Van Den Broek, Marleen H. Schut, Jan Van Herk, and Kees Tuinenbreijer. "Computing emotion awareness through galvanic skin response and facial electromyography." In Probing experience, Springer, Dordrecht, (2008): 149-162. doi:10.1007/978-1-4020-6593-4_14.

[26] Hui, Terence KL, and R. Simon Sherratt. "Coverage of emotion recognition for common wearable biosensors." Biosensors 8 , no. 2 (2018): 30. doi:10.3390/bios8020030.

[27] Cheetham, Marcus, Cátia Cepeda, Hugo Gamboa, James Gilbert, Haim Azhari, and Ali Hesham. "Automated Detection of Mind Wandering: A Mobile Application." BIOSIGNALS 2016 (2016): 198-205. doi:10.5220/0005702401980205.

[28] Al-shargie F. M., T. B. Tang, N. Badruddin, and M. Kiguchi. "Mental Stress Quantification Using EEG Signals.” International Conference for Innovation in Biomedical Engineering and Life Sciences (December 18, 2015): 15-19. doi:10.1007/978-98110-0266-3_4.

[29] Hosseini, Seyyed Abed, and Mohammad Bagher Naghibi-Sistani. "Classification of emotional stress using brain activity." Applied Biomedical Engineering 7 (2011): 32-41. doi:10.5772/18294.

[30] Jena, Sunil. "Examination Stress and Its Effect on EEG.” International Journal of Medical Science and Public Health 4, no. 11 (2015): 1493. doi:10.5455/ijmsph.2015.23042015308.

[31] Hou, Xiyuan, Yisi Liu, Olga Sourina, and Wolfgang Mueller-Wittig. "CogniMeter: EEG-Based Emotion, Mental Workload and Stress Visual Monitoring.” 2015 International Conference on Cyberworlds (CW) (October 2015): 153-160. doi:10.1109/cw.2015.58.

[32] Subhani, Ahmad Rauf, Likun Xia, and Aamir Saeed Malik. "EEG signals to measure mental stress." In 2nd International Conference on Behavioral, Cognitive and Psychological Sciences, BCPS, Maldives (2011): 84-88.

[33] Kalas, Mamta S., and B.F. Momin. "Stress Detection and Reduction Using EEG Signals.” 2016 International Conference on Electrical, Electronics, and Optimization Techniques (ICEEOT) (March 2016): 471-475. doi:10.1109/iceeot.2016.7755604.

[34] Jebelli, Houtan, Sungjoo Hwang, and Sang Hyun Lee. 2018. "EEG-Based Workers' Stress Recognition at Construction Sites.” Automation in Construction 93 (May): 315-24. https://doi.org/10.1016/j.autcon.2018.05.027.

[35] Arsalan, Aamir, Muhammad Majid, Amna Rauf Butt, and Syed Muhammad Anwar. "Classification of perceived mental stress using a commercially available EEG headband." IEEE journal of biomedical and health informatics 23, no. 6 (2019): $2257-2264$. doi:10.1109/JBHI.2019.2926407.

[36] Sun, Feng-Tso, Cynthia Kuo, Heng-Tze Cheng, Senaka Buthpitiya, Patricia Collins, and Martin Griss. "Activity-Aware Mental Stress Detection Using Physiological Sensors." Mobile Computing, Applications, and Services (2012): $282-301$. doi:10.1007/978-3-642-29336-8_16.

[37] Shaffer, Fred, and J. P. Ginsberg. "An Overview of Heart Rate Variability Metrics and Norms.” Frontiers in Public Health 5 (September 28, 2017): 1-17. doi:10.3389/fpubh.2017.00258.

[38] Kleiger, Robert E., Phyllis K. Stein, and J. Thomas Bigger. "Heart Rate Variability: Measurement and Clinical Utility.” Annals of Noninvasive Electrocardiology 10, no. 1 (January 2005): 88-101. doi:10.1111/j.1542-474x.2005.10101.x.

[39] Fernandes de Godoy, Moacir. "Nonlinear Analysis of Heart Rate Variability: A Comprehensive Review.” Journal of Cardiology and Therapy 3, no. 3 (2016): 528-533. doi:10.17554/j.issn.2309-6861.2016.03.101-4.

[40] Järvelin-Pasanen, Susanna, Sanna Sinikallio, and Mika P. Tarvainen. "Heart Rate Variability and Occupational Stresssystematic Review.” Industrial Health 56, no. 6 (2018): 500-511. doi:10.2486/indhealth.2017-0190.

[41] Kim, Hye-Geum, Eun-Jin Cheon, Dai-Seg Bai, Young Hwan Lee, and Bon-Hoon Koo. "Stress and Heart Rate Variability: A Meta-Analysis and Review of the Literature." Psychiatry Investigation 15, no. 3 (March 25, 2018): $235-245$. doi:10.30773/pi.2017.08.17.

[42] Rodríguez-Liñares, Leandro, X. Vila, A. Mendez, M. Lado, and D. Olivieri. "RHRV: An R-based software package for heart rate variability analysis of ECG recordings." In 3rd Iberian conference in systems and information technologies (CISTI 2008): 565-574. 
[43] Boucsein, Wolfram, Don C. Fowles, Sverre Grimnes, Gershon Ben-Shakhar, Walton T. Roth, Michael E. Dawson, and Diane L. Filion. "Publication Recommendations for Electrodermal Measurements." Psychophysiology 49, no. 8 (June 8, 2012): 10171034. doi:10.1111/j.1469-8986.2012.01384.x.

[44] Posada-Quintero, Hugo F. "Electrodermal Activity: What it can Contribute to the Assessment of the Autonomic Nervous System." Doctoral Dissertations, University of Connecticut, United States, (2016).

[45] Lee, Heera, and Andrea Kleinsmith. "Public Speaking Anxiety in a Real Classroom.” Extended Abstracts of the 2019 CHI Conference on Human Factors in Computing Systems (May 2, 2019). doi:10.1145/3290607.3312875.

[46] Aqajari, S. Amir Hossein, E. Kasaeyan Naeini, M. Asgari Mehrabadi, S. Labbaf, A. M. Rahmani, and Nikil Dutt. "GSR Analysis for Stress: Development and Validation of an Open Source Tool for Noisy Naturalistic GSR Data." arXiv preprint arXiv:2005.01834 (2020).

[47] Braithwaite, Jason J., Derrick G. Watson, Robert Jones, and Mickey Rowe. "A guide for analysing electrodermal activity (EDA) \& skin conductance responses (SCRs) for psychological experiments." Psychophysiology 49, no. 1 (2013): 1017-1034.

[48] Greco, Alberto, Gaetano Valenza, Antonio Lanata, Enzo Scilingo, and Luca Citi. "CvxEDA: a Convex Optimization Approach to Electrodermal Activity Processing." IEEE Transactions on Biomedical Engineering 63 (4) (2016): 797-804. doi:10.1109/tbme.2015.2474131.

[49] Benedek, Mathias, and Christian Kaernbach. “A Continuous Measure of Phasic Electrodermal Activity.” Journal of Neuroscience Methods 190, no. 1 (June 2010): 80-91. doi:10.1016/j.jneumeth.2010.04.028.

[50] Benedek, Mathias, and Christian Kaernbach. "Decomposition of Skin Conductance Data by Means of Nonnegative Deconvolution.” Psychophysiology 47 (4) (March 2010): 647-658. doi:10.1111/j.1469-8986.2009.00972.x.

[51] Nicolò, Andrea, Carlo Massaroni, and Louis Passfield. "Respiratory Frequency During Exercise: The Neglected Physiological Measure.” Frontiers in Physiology 8 (December 11, 2017): 1-8. doi:10.3389/fphys.2017.00922.

[52] Han, Lu, Qiang Zhang, Xianxiang Chen, Qingyuan Zhan, Ting Yang, and Zhan Zhao. "Detecting Work-Related Stress with a Wearable Device.” Computers in Industry 90 (September 2017): 42-49. doi:10.1016/j.compind.2017.05.004.

[53] Massaroni, Carlo, Andrea Nicolo, Massimo Sacchetti, and Emiliano Schena. "Contactless Methods for Measuring Respiratory Rate: A Review.” IEEE Sensors Journal (2020): 1-1. doi:10.1109/jsen.2020.3023486.

[54] Hernando, Alberto, Jesus Lazaro, Eduardo Gil, Adriana Arza, Jorge Mario Garzon, Raul Lopez-Anton, Concepcion de la Camara, Pablo Laguna, Jordi Aguilo, and Raquel Bailon. "Inclusion of Respiratory Frequency Information in Heart Rate Variability Analysis for Stress Assessment.” IEEE Journal of Biomedical and Health Informatics 20, no. 4 (July 2016): 1016-1025. doi:10.1109/jbhi.2016.2553578.

[55] Harianto, Januar, Nicholas Carey, and Maria Byrne. "respR - An R Package for the Manipulation and Analysis of Respirometry Data." Edited by Samantha Price. Methods in Ecology and Evolution 10, no. 6 (February 20, 2019): 912-920. doi:10.1111/2041210x.13162.

[56] Elsayed, Nelly, Zaghloul Saad, and Magdy Bayoumi. "Brain Computer Interface: EEG Signal Preprocessing Issues and Solutions.” International Journal of Computer Applications 169, no. 3 (July 17, 2017): 12-16. doi:10.5120/ijca2017914621.

[57] Bigdely-Shamlo, Nima, Tim Mullen, Christian Kothe, Kyung-Min Su, and Kay A. Robbins. "The PREP Pipeline: Standardized Preprocessing for Large-Scale EEG Analysis." Frontiers in Neuroinformatics 9 (June 18, 2015): 1-19. doi:10.3389/fninf.2015.00016.

[58] Pedroni, Andreas, Amirreza Bahreini, and Nicolas Langer. "Automagic: Standardized Preprocessing of Big EEG Data." NeuroImage 200 (October 2019): 460-473. doi:10.1016/j.neuroimage.2019.06.046.

[59] Saitis, Charalampos, and Kyriaki Kalimeri. "Multimodal Classification of Stressful Environments in Visually Impaired Mobility Using EEG and Peripheral Biosignals.” IEEE Transactions on Affective Computing 12, no. 1 (January 1, 2021): $203-214$. doi:10.1109/taffc.2018.2866865.

[60] Bao, Forrest Sheng, Xin Liu, and Christina Zhang. "PyEEG: an open source python module for EEG/MEG feature extraction." Computational intelligence and neuroscience 2011 (2011). doi:.1155/2011/406391.

[61] Helwig, Author Nathaniel E, and Maintainer Nathaniel E Helwig. (2018). "Package 'Eegkit"”. Available online: https://cran.rproject.org/web/packages/eegkit/eegkit.pdf (accessed on January 2021).

[62] A.R., Sohara Banu, and Nagaveni V. "Bio-Signal Analysis for StressDetection Using Machine Learning Methods: A Review." 2020 International Conference on Smart Technologies in Computing, Electrical and Electronics (ICSTCEE) (October 9, 2020): 452-458. doi:10.1109/icstcee49637.2020.9277314.

[63] Shatte, Adrian B. R., Delyse M. Hutchinson, and Samantha J. Teague. "Machine Learning in Mental Health: a Scoping Review of Methods and Applications." Psychological Medicine 49, no. 09 (February 12, 2019): 1426-1448. doi:10.1017/s0033291719000151. 
[64] Gradl, Stefan, Markus Wirth, Robert Richer, Nicolas Rohleder, and Bjoern M. Eskofier. "An Overview of the Feasibility of Permanent, Real-Time, Unobtrusive Stress Measurement with Current Wearables." Proceedings of the 13th EAI International Conference on Pervasive Computing Technologies for Healthcare (May 20, 2019). doi:10.1145/3329189.3329233.

[65] Elzeiny, Sami, and Marwa Qaraqe. "Machine Learning Approaches to Automatic Stress Detection: A Review." 2018 IEEE/ACS 15th International Conference on Computer Systems and Applications (AICCSA) (October 2018). doi:10.1109/aiccsa.2018.8612825.

[66] Rizwan, Md Fahim, Rayed Farhad, Farhan Mashuk, Fakhrul Islam, and Mohammad Hasan Imam. "Design of a Biosignal Based Stress Detection System Using Machine Learning Techniques.” 2019 International Conference on Robotics,Electrical and Signal Processing Techniques (ICREST) (January 2019): 364-368. doi:10.1109/icrest.2019.8644259.

[67] Padmaja, B., V. V. Rama Prasad, and K. V. N. Sunitha. "Machine Learning Approach for Stress Detection Using Wireless Physical Activity Tracker." International Journal of Machine Learning and Computing 8, no. 1 (February 2018): 33-38. doi:10.18178/ijmlc.2018.8.1.659.

[68] Pandey, Purnendu Shekhar. "Machine Learning and IoT for Prediction and Detection of Stress." 2017 17th International Conference on Computational Science and Its Applications (ICCSA) (July 2017). doi:10.1109/iccsa.2017.8000018.

[69] Munla, Nermine, Mohamad Khalil, Ahmad Shahin, and Azzam Mourad. "Driver Stress Level Detection Using HRV Analysis." 2015 International Conference on Advances in Biomedical Engineering (ICABME) (September 2015): 61-64. doi:10.1109/icabme.2015.7323251.

[70] Elgendi, Mohamed, and Carlo Menon. "Machine Learning Ranks ECG as an Optimal Wearable Biosignal for Assessing Driving Stress.” IEEE Access 8 (2020): 34362-34374. doi:10.1109/access.2020.2974933.

[71] Smets, Elena, Pierluigi Casale, Ulf Großekathöfer, Bishal Lamichhane, Walter De Raedt, Katleen Bogaerts, Ilse Van Diest, and Chris Van Hoof. "Comparison of Machine Learning Techniques for Psychophysiological Stress Detection." Pervasive Computing Paradigms for Mental Health (2016): 13-22. doi:10.1007/978-3-319-32270-4_2.

[72] Nakashima, Yoshiki, Jonghwa Kim, Simon Flutura, Andreas Seiderer, and Elisabeth André. "Stress Recognition in Daily Work." Pervasive Computing Paradigms for Mental Health (2016): 23-33. doi:10.1007/978-3-319-32270-4_3.

[73] Sriramprakash, S., Vadana D Prasanna, and O.V. Ramana Murthy. "Stress Detection in Working People." Procedia Computer Science 115 (2017): 359-366. doi:10.1016/j.procs.2017.09.090.

[74] Cho, Dongrae, Jinsil Ham, Jooyoung Oh, Jeanho Park, Sayup Kim, Nak-Kyu Lee, and Boreom Lee. "Detection of Stress Levels from Biosignals Measured in Virtual Reality Environments Using a Kernel-Based Extreme Learning Machine.” Sensors 17, no. 10 (October 24, 2017): 2435. doi:10.3390/s17102435.

[75] Minguillon, Jesus, Eduardo Perez, Miguel Lopez-Gordo, Francisco Pelayo, and Maria Sanchez-Carrion. "Portable System for Real-Time Detection of Stress Level.” Sensors 18, no. 8 (August 1, 2018): 2504. doi:10.3390/s18082504.

[76] Lima, Rodrigo, Daniel Osório, and Hugo Gamboa. "Heart Rate Variability and Electrodermal Activity in Mental Stress Aloud: Predicting the Outcome." Proceedings of the 12th International Joint Conference on Biomedical Engineering Systems and Technologies (2019): 42-51. doi:10.5220/0007355200420051.

[77] Radhamani, Rakhi, Nijin Nizar, Dhanush Kumar, Gayathri Suresh Pillai, Lakshmi Swapna Prasad, Sreehari Sudheer Jitha, Midhun Krishna Vannathi Kuniyil, et al. "Computational Analysis of Cortical EEG Biosignals and Neural Dynamics Underlying an Integrated Mind-Body Relaxation Technique.” Procedia Computer Science 171 (2020): 341-349. doi:10.1016/j.procs.2020.04.035.

[78] Siirtola, Pekka. "Continuous Stress Detection Using the Sensors of Commercial Smartwatch.” Adjunct Proceedings of the 2019 ACM International Joint Conference on Pervasive and Ubiquitous Computing and Proceedings of the 2019 ACM International Symposium on Wearable Computers (September 9, 2019): 1198-1201. doi:10.1145/3341162.3344831. 